\title{
Conical nanopore membranes: solvent shaping of nanopores
}

\author{
Paolo Scopece ${ }^{1,2}$, Lane A Baker ${ }^{1}$, Paolo Ugo ${ }^{2}$ and \\ Charles R Martin ${ }^{1,3}$ \\ ${ }^{1}$ Departments of Chemistry and Anesthesiology and Center for Research at the Bio/Nano \\ Interface, University of Florida, Gainesville, FL 32611-7200, USA \\ ${ }^{2}$ Department of Physical Chemistry, University Ca' Foscari of Venice, Dorsoduro 2137, \\ 30123 Venice, Italy \\ E-mail: crmartin@chem.ufl.edu
}

Received 19 May 2006, in final form 22 June 2006

Published 11 July 2006

Online at stacks.iop.org/Nano/17/3951

\begin{abstract}
We have undertaken a systematic investigation of the influence of ethanol on the shape of conical pores produced by the track-etch technique in poly(ethylene terephthalate) films. We have found that the cone angle of the conical nanopore generated is dependent on the amount of ethanol present in an alkaline etching solution. By varying the percentage of ethanol in the etch solution, precise control over the geometry of the conical nanopore and nanomaterials templated within these pores can be attained. We prove this by plating gold nanocones within the various conical nanopores prepared, dissolving the membrane to liberate the nanocones, and imaging the nanocones using scanning electron microscopy. The results of these investigations are reported here.
\end{abstract}

\section{Introduction}

We and others have been investigating a general method for preparing nanomaterials known as template synthesis $[1,2]$. This method entails the synthesis or deposition of a desired material within the pores of a nanopore membrane that serves as a template. These template membranes contain monodisperse pores that are typically cylindrical in geometry, and the pore diameter can be varied at will from tens of nanometres to tens of microns. Since typical pore geometries are cylindrical, correspondingly cylindrical nanostructures are usually synthesized via the template method; depending on the membrane and synthetic method used, these may be solid nanowires or hollow nanotubes [3].

Recently, we and others have become interested in nanopores that have a conical pore shape and the correspondingly conical nanostructures synthesized via the template method within these pores. A number of applications can potentially benefit from a conical pore geometry. For instance, it has been shown that such conically shaped nanopores can be used as the sensing element for new types of small molecule [4], DNA [5-8], protein [9] and

3 Author to whom any correspondence should be addressed. particle [10] sensors. Conically shaped gold nanotubes deposited within such pores can also act as mimics of voltagegated ion channels [11]. Membranes used for separations might also benefit from a highly asymmetric pore structure. Finally, in addition to sensing and separations platforms, conical nanostructures prepared by more conventional methods have been proposed for use as cathodes in field-emission displays [12]. In all of these applications it is important to be able to control the geometry of the conical nanopore or nanostructure; i.e., the cone angle of the conical nanopore.

The importance of such conically shaped nanopores and nanostructures has led us to develop and refine methods for preparing membranes with conical pores. We and others have shown that the well-known track-etch method [13] can be used as a starting place for preparing such conical nanopores [14-20]. This method entails bombarding a thin (5-20 $\mu \mathrm{m})$ film of the polymer with a collimated beam of high-energy particles to create parallel damage tracks through the film. To make cylindrical pores, the tracked membrane is simply immersed into a chemical etch bath, where preferential etching along the damage track converts each track into a cylindrical pore. To make conical pores, the tracked membrane is mounted in an etching cell with an etch solution on one side of the tracked membrane and a stop solution on the other 


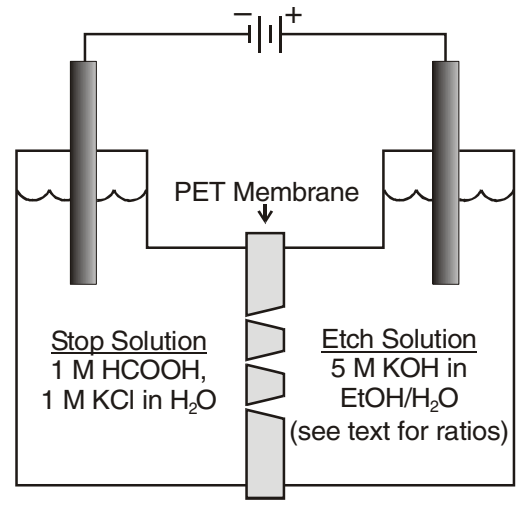

Figure 1. Schematic of an etching cell for preparation of conical nanopore membranes.

side [14]. This is shown schematically in figure 1. Since the damage track is etched faster at the face of the membrane exposed to the etch solution than at the face of the membrane exposed to the stop solution, conically shaped nanopores are obtained.

While this stop-etch approach has been used to make conical nanopores in a variety of different tracked materials, a key issue that remains is fine control over the geometry of the resulting conical nanopore. Recently, we have demonstrated that a transmembrane potential applied across the membrane while etching can be used to control the shape of conical nanopores fabricated in polycarbonate membranes [21]. Dobrev and coworkers [22] showed that varying the hydrostatic pressure on one side of the membrane can also influence the shape of pores fabricated using the track-etch process. A very brief report by Oleinikov et al [23] suggests the cone angle of conical pores etched into poly(ethylene terephthalate) (PET) films can be controlled by varying the chemical composition (ethanol/water ratio) of the etch bath; however, little supporting data are presented in this report. Chtanko and coworkers [24] have also recently reported the preparation of asymmetric nanowires and nanopores in polycarbonate membranes using a similar procedure. In this study, we have undertaken a systematic investigation of this stop-etch chemistry and have found that precise control over pore geometry can be obtained for conical pores etched in PET films. We prove this by plating gold nanocones within the various conical nanocones prepared, dissolving the membrane to liberate the nanocones, and imaging the nanotubes using scanning electron microscopy. The results of these investigations are reported here.

\section{Experimental section}

\subsection{Materials}

The following chemicals and materials were used as received: ethyl alcohol (USP, absolute, 200 proof, AAPER alcohol), $\mathrm{KOH}$ (pellets, ACROS organics), $\mathrm{KCl}$ (certified A.C.S., Fisher Scientific), $\mathrm{HCOOH}$ (88\% in water, Fisher Biotech), $\mathrm{CHCl}_{3}$ (certified A.C.S., Fisher Scientific), 1,1,1,3,3,3-hexafluoro-2propanol (HFIP) $(99+\%$, Aldrich), alumina filters (Anodisc $25 \mathrm{~mm}, 0.1 \mu \mathrm{m}$, Whatman). Ion-tracked PET films $(12 \mu \mathrm{m}$

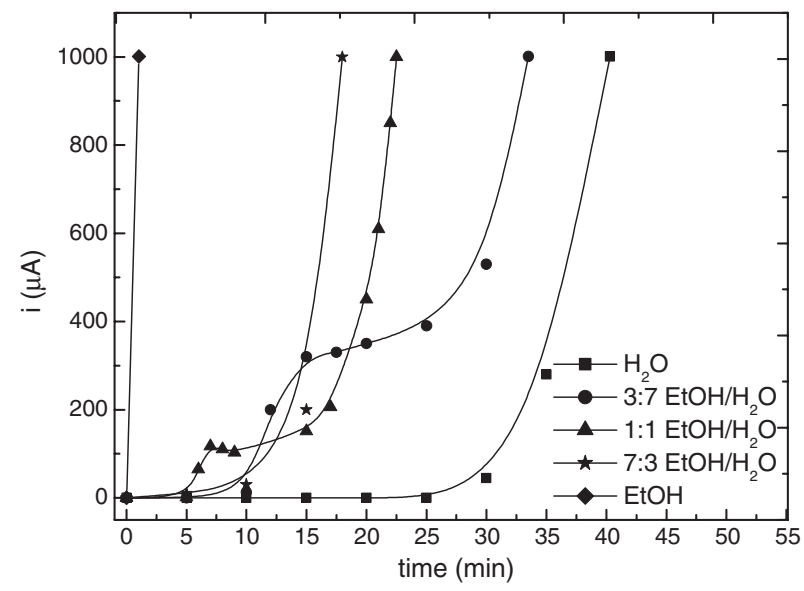

Figure 2. Plot of current versus time for each composition of each etch solution used: (1) water, (2) 3:7 ethanol/water, (3) 1:1 ethanol/water, (4) 7:3 ethanol/water, and (5) ethanol. All solutions were $5 \mathrm{M} \mathrm{KOH}$. Applied transmembrane potential $=30 \mathrm{~V}$; pore density $=10^{6}$ pores $\mathrm{cm}^{-2} ;$ membrane area $=0.5 \mathrm{~cm}^{2}$.

thick, $10^{6}$ tracks $\mathrm{cm}^{-2}$ ) were obtained from GSI, Darmstadt, Germany.

\subsection{Chemical etching of conical nanopores}

The procedure we have used to prepare conical pores is based on the work of Apel et al [14]. In order to obtain constant etching parameters, oxidation of the damage track was saturated by exposing tracked membranes to UV irradiation ( $1 \mathrm{~h}$ exposure time for each side). Iontracked poly(ethylene terephthalate) films were mounted in a tape mask with a well-defined circular hole, as described previously [15]. Asymmetric chemical etching was performed at room temperature by mounting the tape-masked membrane between two halves of a glass U-tube permeation cell (figure 1). One side of the cell (hereafter referred to as the base side of the membrane) was filled with an etch solution of $5 \mathrm{M} \mathrm{KOH}$ in variable ethanol/water ratios (as described in the results), and the other side (hereafter referred to as the tip side of the membrane) was filled with a stopping medium of $1 \mathrm{M}$ $\mathrm{KCl}$ and $1 \mathrm{M} \mathrm{HCOOH}$. A $30 \mathrm{~V}$ potential was applied across the membrane by two Pt electrodes and the electrical current was monitored with a Keithley 6487 picoammeter/voltage source. The electrode in the etch solution served as the anode, and the electrode in the stop solution served as the cathode. The current was initially zero but increased to finite values when the etchant had broken through to the stop solution on the other side of the membranes (figure 2). Etching was stopped when a current of $1 \mathrm{~mA}$ was reached, and the membrane was immediately immersed (both sides) in stop medium for $30 \mathrm{~min}$ to neutralize residual base. The etched membrane was then soaked for at least $1 \mathrm{~h}$ in $18 \mathrm{M} \Omega$ water to remove residual salts.

\subsection{Electroless gold plating of conical nanopores}

Template synthesis via the electroless deposition of gold was performed as previously described $[1,2]$. To prepare the nanomaterials described here, plating was performed overnight in order to fill the pores and obtain complete coverage of all 
surfaces. Electroless plating causes a gold nanocone to be deposited in each pore; in addition, both faces of the membrane are coated with thin films of gold.

\subsection{Liberating the gold nanocones}

To obtain suspensions of the gold nanocones, the gold surface films on both faces of the membrane were removed by applying and then removing a piece of Scotch tape (810), and the membrane was cut from the original tape mask. The membrane was then immersed in a solution of $\mathrm{HFIP} / \mathrm{CHCl}_{3}(1: 9$ by vol) for $30 \mathrm{~min}$. This results in dissolution of the membrane thus liberating the template synthesized gold nanocones. The nanocones were collected from solution by filtration on an anodisc filter. The anodisc filter and filtrate were then coated with $\mathrm{Au} / \mathrm{Pd}$ using a Denton Vacuum Desk II sputtering system. Electron micrographs were obtained using a Hitachi S4000 or a JEOL 6335F scanning electron microscope (SEM). Measurements of cone length, cone angle and base diameter were performed using Image $\mathbf{J}$ software.

\subsection{Obtaining arrays of standing gold nanocones}

Gold-plated conical nanopore membranes were rinsed with $18 \mathrm{M} \Omega$ water and dried under flowing $\mathrm{N}_{2}$. The gold surface film on one face of the membrane was tape peeled away. The freshly exposed face of the membrane was then subjected to an oxygen plasma-etch to remove the membrane template using a Samco plasma reactive ion etching system (model RIE-1C). Electron micrographs of the resulting nanocone array were obtained as described above.

\section{Results/discussion}

During chemical etching of ion-tracked membranes, the damaged zone of a latent track is transformed into a nanopore [13]. The simplest description of the etching process defines two parameters: the bulk etch rate $\left(V_{\mathrm{B}}\right)$ and the track etch rate $\left(V_{\mathrm{T}}\right) . V_{\mathrm{B}}$ depends on material, etchant composition and temperature. $V_{\mathrm{T}}$ depends on additional parameters, such as sensitivity of the material to tracking, post-irradiation conditions and etching conditions. To obtain conical pores in a tracked PET membrane, we have increased the $V_{\mathrm{B}} / V_{\mathrm{T}}$ ratio by modifying the etch solution composition with different concentrations of ethanol. The effect of alcohol addition, together with the high applied transmembrane potential $(30 \mathrm{~V})$, leads to conically shaped pores that etch very quickly compared to an aqueous etchant at $1 \mathrm{~V}$ applied transmembrane potential (common etching conditions) $[9,14,19]$.

A comparison of the effect of solvent on the time required to reach $1 \mathrm{~mA}$ of total current using $5 \mathrm{M} \mathrm{KOH}$ as the etchant is shown in figure 2. The solvents used were water, 3:7 ethanol/water (numbers indicate the solvent ratio by volume), 1:1 ethanol/water, 7:3 ethanol/water, and ethanol. At the same temperature, applied potential, and concentration of base, we have found that increasing the amount of ethanol progressively decreases the time required to reach $1 \mathrm{~mA}$ current. For example, an aqueous alkali solution requires $40 \mathrm{~min}$ to reach $1 \mathrm{~mA}$ of current. With an ethanolic alkali solution $1 \mathrm{~mA}$ current is reached in about $1 \mathrm{~min}$. Since PET membranes are

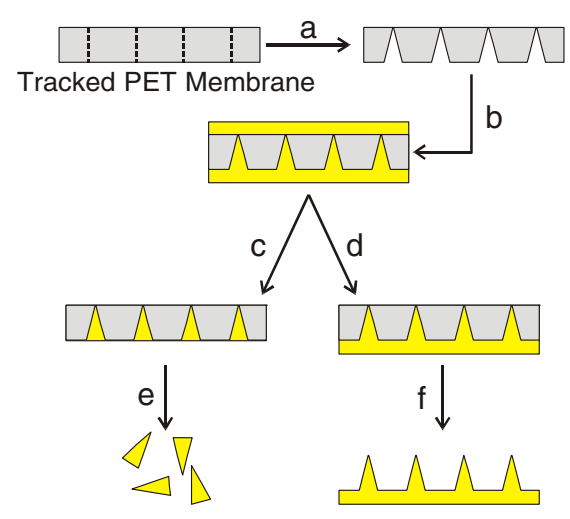

Figure 3. Schematic diagram of the method used to prepare templated Au nanocones: (a) asymmetric etch, (b) electroless Au plate, (c) double sided tape-strip, (e) dissolution of the membrane and filtration of nanocones, (d) front side tape-strip, (f) oxygen plasma etch of the front side of the membrane.

(This figure is in colour only in the electronic version)

moderately hydrophobic, we believe the effect of alcohol is to increase solvation of the polymer chains in the membrane. This makes the ester linkages in the polymer backbone more accessible to cleavage by the basic etching solution.

\subsection{Template synthesis of nanocones}

To determine the three-dimensional structure of the conical nanopore, the pores were filled with gold and the membrane removed, producing a replica of the empty volume of the conical nanopore. These nanopore replicas were obtained by using an electroless plating method to deposit a gold nanocone within each pore of the membrane $[17,21]$. As shown schematically in figure 3 , this method yields gold nanocones within the pores of the membrane and gold surface layers that coat both faces of the membrane.

To liberate nanocones the left branch of figure 3 is followed. First (step a), conical pores are etched in a PET membrane. Second (step b), the entire membrane is subjected to electroless deposition of Au. Third (step c), the front and back layers of $\mathrm{Au}$ are removed with tape, leaving $\mathrm{Au}$ deposited in the conical pores of the membrane. The back (cone base) of a tape-peeled conical nanopore membrane is shown in figure 4. Finally (step e), the nanocones are liberated by dissolving the membrane in hexafluro isopropanol to yield a suspension of the gold nanocones. This suspension was filtered through an alumina filter, and the nanocones on the filter surface were imaged using field-emission scanning electron microscopy (SEM).

Representative SEM images of each etching solution composition are shown in figure 5. The volume fraction of ethanol present in the etching solution increases from left to right and from top to bottom in this series of images. It is obvious from these images that as the per cent ethanol increases in the etch solution, the cone angle of the templated $\mathrm{Au}$ nanocone increases, as does the original template conical nanopore. A number of $\mathrm{Au}$ nanocones were measured and tabulated for each etching condition; the results are discussed further below. 


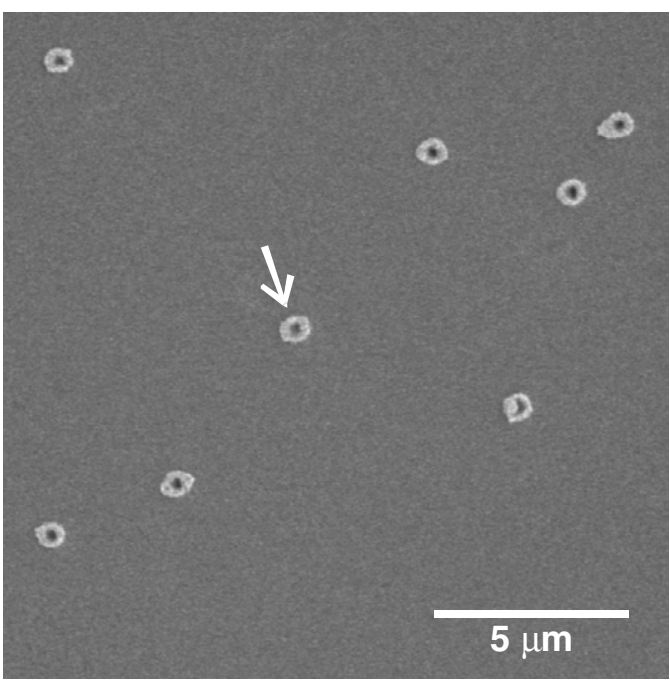

Figure 4. SEM image of the base side of a tape-stripped conical nanopore membrane.
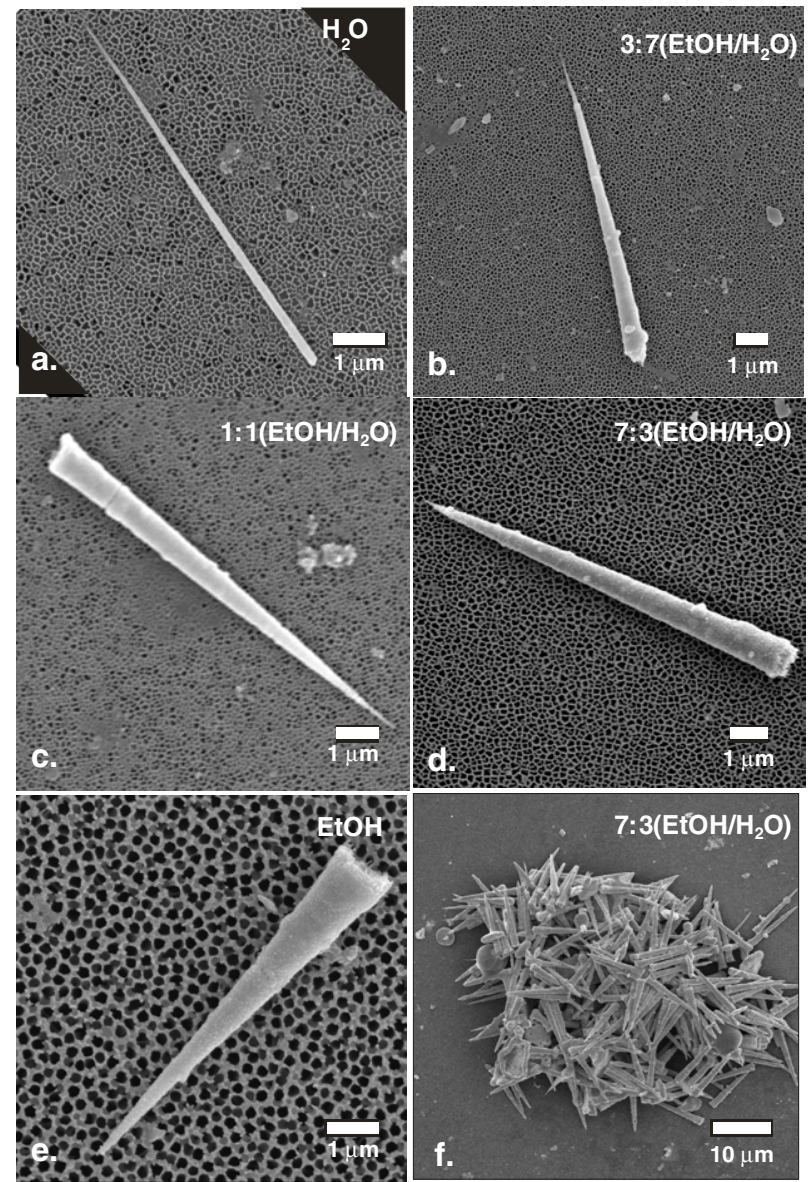

Figure 5. SEM images of liberated Au nanocones after filtration showing the effect of the volume fraction of ethanol in the etch solution. (a) water, (b) 3:7 ethanol/water, (c) 1:1 ethanol/water, (d) 7:3 ethanol/water, and (e) ethanol, (f) aggregation of nanocones after modification with a fluorinated thiol.

An alternative to liberated nanocones-arrays of standing $\mathrm{Au}$ nanocones-can be generated by slight modifications in

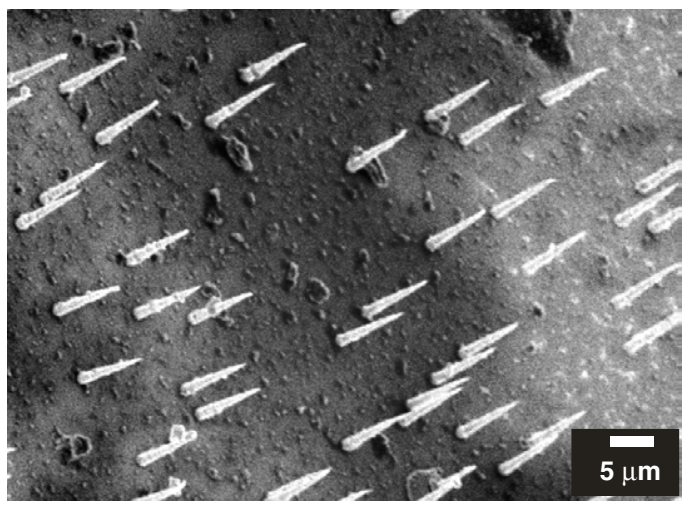

Figure 6. SEM image of standing Au nanocone array obtained after oxygen plasma etch (figure 5 , step f).

Table 1. Comparison of cone geometries obtained using different ratios of ethanol/water in the etch solution.

\begin{tabular}{lccccc}
\hline $\begin{array}{l}5 \mathrm{M} \mathrm{KOH} \\
\text { etch } \\
\text { solution }\end{array}$ & $\begin{array}{l}\text { Length } \\
(\mu \mathrm{m})\end{array}$ & $\begin{array}{l}\text { Base width } \\
(\mathrm{nm})\end{array}$ & $\begin{array}{l}\text { Cone } \\
\text { half- } \\
\text { angle } \\
\theta(\mathrm{deg})\end{array}$ & $\begin{array}{l}\text { Time } \\
\text { to } \\
\text { etch } \\
(\mathrm{min})\end{array}$ & $\begin{array}{l}\text { Pore } \\
\text { resistance } \\
(\mathrm{M} \Omega)\end{array}$ \\
\hline $\mathrm{H}_{2} \mathrm{O}$ & $7.4 \pm 0.9$ & $190 \pm 20$ & $0.5 \pm 0.1$ & 41 & 690 \\
$3: 7$ & & & & & \\
$\mathrm{EtOH} / \mathrm{H}_{2} \mathrm{O}$ & $9.1 \pm 0.6$ & $720 \pm 40$ & $1.8 \pm 0.2$ & 35 & 200 \\
$1: 1$ & & & & & \\
$\mathrm{EtOH} / \mathrm{H}_{2} \mathrm{O}$ & $9.2 \pm 0.7$ & $820 \pm 40$ & $2.3 \pm 0.2$ & 22 & 160 \\
$7: 3$ & & & & & \\
$\mathrm{EtOH} / \mathrm{H}_{2} \mathrm{O}$ & $10.2 \pm 0.6$ & $840 \pm 50$ & $2.5 \pm 0.2$ & 18 & 140 \\
$\mathrm{EtOH}$ & $7.5 \pm 0.7$ & $1270 \pm 110$ & $4.4 \pm 0.5$ & 2 & 82 \\
\hline
\end{tabular}

${ }^{\mathrm{a}}$ Measured from SEM image.

${ }^{\mathrm{b}}$ Calculated for each measured cone angle by applying equation (3), and assuming a nanopore tip diameter of $10 \mathrm{~nm}$, a membrane thickness of $10 \mu \mathrm{m}$, and an electrolyte with resistivity of $0.1 \Omega \mathrm{m}$ (see text for details).

the template synthesis procedure. A schematic summary of this process is shown in the right branch of figure 3. First, we perform the initial two steps ( $a$ and $b$ ) as described above to prepare conical nanopores and templated $\mathrm{Au}$ nanocones. Next (step d), the Au surface layer that faced the stop solution (tip side of the membrane) is removed. The membrane is then affixed to a SEM stub to facilitate subsequent analysis. The polymer template is then removed (step f) from the random nanocone array by reactive ion etching in an oxygen plasma $[17,21]$. This yields an array of cones sitting on their bases. A SEM image of one of these nanocone ensembles is shown in figure 6 .

\subsection{Conical nanopore structure}

In table 1, a comparison of the geometries measured from SEM images of nanocones obtained under different etching conditions is presented. In figure 5 , representative electron micrographs are shown. In all cases a conical geometry is obtained. Changing the alkali etch solvent from pure water (figure 5(a)) to pure ethanol (figure 5(e)) results in a progressively more obtuse cone angle. For intermediate ethanol concentrations a less dramatic, but observable, difference is attained. 


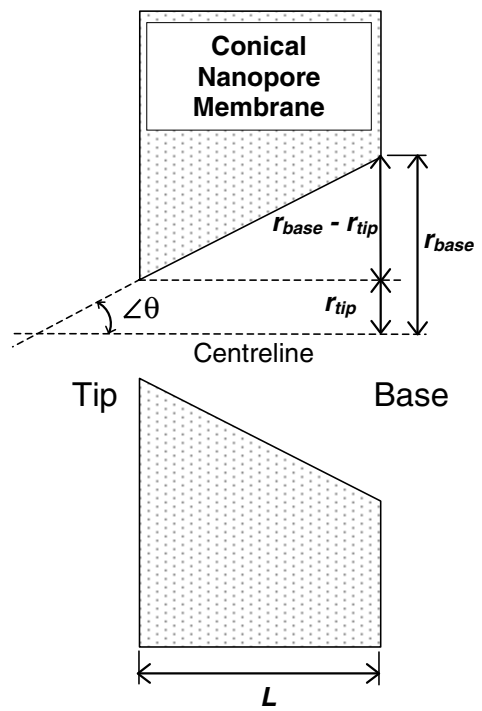

Figure 7. Diagram of a conical nanopore with notations for parameters of interest.

Several observations can be made. First, increasing the ethanol content of the etch solution results in an increased cone angle, which in turn results in an increased base diameter for the nanocone. This asymmetry in etch, as represented by cone half-angle, increases from the $0.5^{\circ}$ in the case of pure water, to $4.4^{\circ}$ in the case of pure ethanol solvents. The significance of control over the cone angle can be illustrated by considering the effect the cone angle plays in the resistance of an electrolyte-filled conical nanopore. The resistance for a conical pore $(R)$ can be calculated based on the following equation [17]:

$$
R=\frac{4 \rho L}{\pi d_{\text {tip }} d_{\text {base }}}
$$

where $\rho$ is the resistivity of the electrolyte, $L$ is the length of the pore, and $d_{\text {base }}$ and $d_{\text {tip }}$ are the diameter at the base and tip of the cone, respectively. Substituting $2 r_{\text {tip }}$ for $d_{\text {tip }}$ and $2 r_{\text {base }}$ for $d_{\text {base }}$ gives:

$$
R=\frac{\rho L}{\pi r_{\text {tip }} r_{\text {base }}} .
$$

A diagram of a conical pore with these parameters labelled is shown in figure 7 (not to scale). The quantity $r_{\text {base }}$ is calculated by summing $r_{\text {tip }}$ and the difference of the base and the tip $\left(r_{\text {base }}-r_{\text {tip }}\right)$, which is equal to the quantity $(L \tan \theta)$, where $\theta$ is the cone half-angle. Thus the influence of cone angle on the resistance for a conical nanopore of fixed tip radius and membrane thickness is given by the following equation:

$$
R=\frac{\rho L}{\pi r_{\text {tip }}\left(r_{\text {tip }}+L \tan \theta\right)}
$$

To quantify the effect of the cone angle on the resistance of the conical nanopore of a given diameter, we will fix $r_{\text {tip }}$ at $5 \mathrm{~nm}, L$ at $10 \mu \mathrm{m}$, and the solution resistivity $(\rho)$ at $0.1 \Omega \mathrm{m}$. These are reasonable values, as typically the nanopore tip is selected to match the size of the analyte of interest in a sensing or separations application, and proteins typically have radii of the order of $2-3 \mathrm{~nm}$. Inputting the measured cone angles for each composition of etch solution, then yields the calculated pore resistances given in table 1 . As can be observed, the calculated ohmic resistance for a conical nanopore of identical tip size and membrane thickness is nearly an order of magnitude lower if the cone angle is increased from $0.5^{\circ}$ to $4.4^{\circ}$. (The cone angles obtained for pores prepared using pure water and pure ethanol as solvents, respectively.) Thus, the cone angle of the conical nanopore is, based on the discussion above, obviously a critical parameter that influences membrane performance for a variety of applications. These applications include sensing and separations, as the transport properties of conical pores differ markedly from cylindrical pores.

A second observation - conical nanomaterials of uniform shape and size can be template synthesized using these conical nanopore membranes-can also be drawn from the data presented. In addition to the statistical data presented in table 1 , in figure 5 , panel $\mathrm{f}$, an aggregate of template synthesized Au nanocones is shown. Aggregation was induced by modifying the surface of the nanocones with a fluorinated thiol. In an aqueous suspension, aggregation is promoted because the fluorinated thiol coating the nanocone makes the nanocone hydrophobic. (In the absence of a fluorinated thiol coating on the nanocones, no aggregation is observed.) The ability to synthesize nanomaterials with unique shapes and sizes (i.e. rods, spheres, prisms) has proved important for applications ranging from sensing to self-assembly [25]. We expect such conical nanostructures to contribute to the experimental nanotechnologists toolbox as well.

\section{Conclusions}

In this paper we have described a systematic investigation of the influence of ethanol on the shape of conical pores produced by the track-etch technique in poly(ethylene terephthalate) films. We have found that the cone angle of the conical nanopore generated is dependent on the volume fraction of ethanol present in an alkaline etching solution. By varying the percentage of ethanol in the etch solution, precise control over the geometry of the conical nanopore and nanomaterials templated within these pores can be attained. By plating gold nanocones within the various conical nanopores prepared, dissolving the membrane to liberate the nanocones, and imaging the nanocones using scanning electron microscopy the shape and structure of these pores has been demonstrated.

Future work is aimed at exaggerating the cone angles obtained and further controlling the nanoscale structure of the porous membranes. We believe such membranes will find diverse applications in nanotechnology, including template synthesis, separations, and chemical sensing.

\section{Acknowledgments}

We would like to thank Dr Zuzanna Siwy and Dr C Chad Harrell for helpful discussions. This work was supported by the Air Force Office of Scientific Research and the National Institutes of Health. 


\section{References}

[1] Martin C R 1994 Science 266 1961-6

[2] Martin C R 1996 Chem. Mater. 8 1739-46

[3] Kovtyukhova N I and Mallouk T E 2002 Chem. Eur. J. 8 4354-63

[4] Heins E A, Siwy Z S, Baker L A and Martin C R 2005 Nano Lett. 5 1824-9

[5] Harrell C C, Choi Y, Baker L A, Siwy Z S and Martin C R 2006 Langmuir submitted

[6] Harrell C C, Kohli P, Siwy Z and Martin C R 2004 J. Am Chem. Soc. 126 15646-7

[7] Mara A, Siwy Z, Trautmann C, Wan J and Kamme F 2004 Nano Lett. 4 497-501

[8] Schiedt B, Healy K, Morrison A P, Neumann R and Siwy Z 2005 Nucl. Instrum. Methods Phys. Res. B 236 109-16

[9] Siwy Z, Trofin L, Kohli P, Baker L A, Trautmann C and Martin C R 2005 J. Am. Chem. Soc. 127 5000-1

[10] Lee S, Zhang Y, White H S, Harrell C C and Martin C R 2004 Anal. Chem. 76 6108-15

[11] Siwy Z S 2006 Adv. Funct. Mater. 16 735-46

[12] Merkulov V I, Guillorn M A, Lowdnes D H, Simpson M L and Voelkl E 2001 Appl. Phys. Lett. 79 1178-80

[13] Fleischer R L, Price P B and Walker R M 1975 Nuclear Tracks in Solids. Principles and Applications (Berkeley, CA: University of California Press)
[14] Apel P Y, Korchev Y E, Siwy Z, Spohr R and Yoshida M 200 Nucl. Instrum. Methods Phys. Res. B 184 337-46

[15] Harrell C C, Lee S B and Martin C R 2003 Anal. Chem. 75 6861-7

[16] Heins E A, Baker L A, Siwy Z S, Mota M O and Martin C R 2005 J. Phys. Chem. B 109 18400-7

[17] Li N, Yu S, Harrell C C and Martin C R 2004 Anal. Chem. 76 2025-30

[18] Siwy Z, Apel P, Baur D, Dobrev D D, Korchev Y E, Neumann R, Spohr R, Trautmann C and Voss K-O 2003 Surf. Sci. 532-535 1061-6

[19] Siwy Z, Heins E, Harrell C C, Kohli P and Martin C R 2004 J. Am. Chem. Soc. 126 10850-1

[20] Siwy Z, Kosinska I D, Fulinski A and Martin C R 2005 Phys. Rev. Lett. 94048102

[21] Harrell C C, Siwy Z S and Martin C R 2006 Small 2 194-8

[22] Dobrev D, Vetter J, Nuemann R and Angert N 2001 J. Vac. Sci. Technol. B 19 1385-7

[23] Oleinikov V A, Tolmachyova Y V, Berezkin V V, Vilensky A I and Mchedlishvili B V 1995 Radiat. Meas. $25713-4$

[24] Chtanko N, Molares M E T, Cornelius T, Dobrev D and Neumann R 2004 J. Phys. Chem. B 108 9950-4

[25] Im S H, Lee Y T, Wiley B and Xia Y 2005 Angew. Chem. Int $E d .442154-7$ and references cited therein 